http://jmscr.igmpublication.org/home/

ISSN (e)-2347-176x ISSN (p) 2455-0450

crossref DOI: https://dx.doi.org/10.18535/jmscr/v8i6.45

Journal Of Medical Science And Clinical Research

\title{
Maternal and perinatal outcome in severe preeclampsia and eclampsia
}

\author{
Authors \\ Dr Rajni Priyanka ${ }^{*}$, Dr Chitra Sinha ${ }^{2}$ \\ ${ }^{1}$ Senior Resident, Dept. of Obst \& Gynae, P.M.C.H, Patna \\ ${ }^{2}$ Associate Professor, Dept. of Obst \& Gynae, P.M.C.H, Patna \\ *Corresponding Author \\ Dr Rajni Priyanka \\ Senior Resident, Dept. of Obst \& Gynae, P.M.C.H, Patna, India
}

\begin{abstract}
Background: Preeclampsia and eclampsia is one of the leading causes of maternal and perinatal morbidity and mortality. It is an acute life endangering complication of pregnancy.

Aim: The objective of the present study was to study the maternal and fetal outcome in patients with severe preeclampsia and eclampsia in a tertiary care centre.

Study Design: Prospective Study

Study Period: From January 2019 to December 2019 over a period of one year.

Material and Method: Total 140 women with severe preeclampsia and eclampsia were included in the study according to the inclusion and exclusion criteria, who were admitted in obstetric emergency unit of Patna medical college hospital over a period of one year.

Results: In present study $77.85 \%$ cases were unbooked and referred to this centre. Out of 140, 91 patients $(65 \%)$ are between 20-30 year of age group. Majority of patients 75 (53.57\%) were primigravida. Majority of patient were preterm 96(68.57\%). Out of 140 patients, 87 (62.14\%) patients were referred for convulsions and $45.71 \%$ with high blood pressure. Cesarean section was done in $49.28 \%$ patient for failed induction (46.3\%) or fetal distress (24.7\%).vaginal delivery occured in $38.57 \%$. The mean delivery was 6.8 hours. Maternal complications included PPH in 51, abruption placenta in 11 and DIC in 19. Out of 140 cases, 106 were live birth, 24 intrauterine deaths, and 12 still births. Low birth weight babies were $47.85 \%$ and NICU admission required in $59.28 \%$ cases. There were $10(7.14 \%)$ cases of maternal death. perinatal loss was $34.28 \%$.
\end{abstract}

Conclusion: A Proper antenatal care, early intervention and prompt treatment is required to reduce maternal and perinatal outcome in patients with severe preeclampsia and eclampsia.

Keywords: obstetric outcome, eclampsia, severe preeclampsia, maternal morbidity, perinatal morbidity.

\section{Introduction}

Preeclampsia / eclampsia is one of the $3^{\text {rd }}$ leading cause of maternal morbidity and mortality worldwide and accounts for $14 \%$ maternal death ${ }^{1}$. Maternal complications and mortality due to preeclampsia /eclampsia in developing countries is very high and mainly due to lack of access to healthcare facility, lack of resources, inappropriate diagnosis and delayed referral to higher centre ${ }^{2}$. So a clear protocol for early detection and timely care is required for better maternal and perinatal outcome. 
WHO estimated that approximately 60,000 women die each year from preeclampsia worldwide. Preeclampsia and eclampsia account for $24 \%$ of all maternal death in India. The incidence of Preeclampsia and eclampsia in India is around $4.6 \%$ and the neonatal mortality rate is 41 per 1000 live birth ${ }^{3}$.

Preeclampsia is a pregnancy specific syndrome of vasospasm and endothelial activation throughout maternal circulation leading to reduced organ perfusion with multiorgan dysfunction ${ }^{4}$.

Eclampsia is defined as the new onset of generilized tonic clonic seizure in a women with severe preeclampsia. Seizures occur most commonly in postnatal period in $44 \%$ cases, $38 \%$ in antenatal period and in $18 \%$ cases during intrapartum period ${ }^{5}$.

Eclampsia and severe preeclampsia are associated with complications like placental abruption, disseminated intravascular coagulation pulmonary edema, cardiac failure, HELLP syndrome, renal failure, aspiration pneumonitis and cerebral hemorrhage, which are major attributable factor for maternal death ${ }^{6}$. The incidence of eclampsia can be reduced by better antenatal care, early detection of the disorder and prompt treatment.

The main treatment option is to deliver the patient .Time interval from admission to delivery is a major factor influencing maternal and fetal outcome. Its always have to make a balance between mother and fetus according to the maternal condition, however delivery is not always in best interest of fetus.

The incidence of eclampsia can be reduced by better antenatal care, early detection of the disorder and prompt treatment.

\section{Material and method -}

The present study is a prospective study on 140pregnant women of severe preeclampsia and eclampsia who were admitted in emergency obstetric unit of Patna medical college hospital from January 2019 to December 2019, over a period of one year.
Inclusion Criteria- women with severe preeclampsia/ eclampsia were included in this study.

Exclusion Criteria- Women with medical complications like anaemia, chronic hypertension, diabetes, vascular and renal disease, multiple pregnancy, polyhydramnios, were excluded from the study.

On admission, detailed demographic, personal, medical, obstetric and family history was taken from the patient or her attended. General physical examination, systemic, abdominal and pelvic examinations were done. Blood investigations including complete blood picture with platelet count, coagulation profile, liver function test, renal function test were sent. Urine routine microscopy was done especially to see protenuria. After assessment and stabilization of the maternal status, fetal condition was evaluated with the help of FHR recording, CTG tracing and ultrasound.

The decision regarding timing and mode of delivery were individualized. Corticosteroids were administered if gestational age was less than 34 weeks gestation. Magnesium sulphate were given in eclamptic patients and in patient with severe preeclampsia with imminent sign of eclampsia. Antihypertensive drugs labetalol, nifedepin were used to control blood pressure. Obstetric management was done according to the maternal and fetal condition. The decision to deliver the patient vaginally (either spontaneous or induced) or by caesarean section was individualised and decided as per the hospital protocol and emergency medical team. The patient with uncontrolled hypertension were managed with the help of physician and anesthetist. The outcome of each pregnancy was obtained by examining the patient in labour ward, neonatal care was provided by paediatrician from delivery onwards.

The variables include age, date and time of admission, gestational age, mode and time of delivery, Apgar score, birth weight, time interval between admission and delivery and maternal and fetal morbidity and mortality. 


\section{Result}

Table 1 Demographic features, Age, Parity \& Gestational age

\begin{tabular}{|l|c|c|}
\hline Booking status & Number & Percentage \\
\hline Unbooked & 109 & $77.85 \%$ \\
\hline Booked & 31 & $22.14 \%$ \\
\hline Age group(year) \\
\hline$<20$ & 21 & $15 \%$ \\
\hline $21-25$ & 63 & $39.28 \%$ \\
\hline $26-30$ & 28 & $12.85 \%$ \\
\hline $31-35$ & 16 & $10.71 \%$ \\
\hline More than 35 & 12 & $8.57 \%$ \\
\hline Parity & 75 & $53.57 \%$ \\
\hline 1 & 21 & $15 \%$ \\
\hline 2 & 11 & $7.85 \%$ \\
\hline 3 & 33 & $23.57 \%$ \\
\hline More than 3 & 11 & $7.85 \%$ \\
\hline Gestational age (weeks) & $19.28 \%$ \\
\hline$<28$ & 27 & $41.42 \%$ \\
\hline $29-32$ & 58 & $24.28 \%$ \\
\hline $32-36$ & 34 & $7.14 \%$ \\
\hline $37-40$ & 10 & \\
\hline$>40$ & \multicolumn{2}{|l|}{} \\
\hline
\end{tabular}

In present study $77.85 \%$ cases were unbooked and referred to this centre. 91 patients $(65 \%)$ are between 20 - 30 year of age group, followed by $15 \%$ of patients are less than 20 year. Majority of patients $75(53.57 \%)$ were primigravida and, 33(23.57\%) were grandmultipara.

Majority of patient were preterm 96(68.57\%) presented before 37 completed weeks. $24.28 \%$ were term and $7.14 \%$ were post-dated.

Table 2Distribution of patient according to presenting symptoms

\begin{tabular}{|l|c|c|}
\hline Symptoms & $\begin{array}{c}\text { Number } \\
\text { of patient }\end{array}$ & Percentage \\
\hline convulsion & 87 & $62.14 \%$ \\
\hline High blood pressure & 64 & $45.71 \%$ \\
\hline Headache & 45 & $32.14 \%$ \\
\hline Blurring of vision & 16 & $11.42 \%$ \\
\hline Epigastric pain & 13 & $9.28 \%$ \\
\hline Generalised edema & 17 & $12.14 \%$ \\
\hline oliguria & 13 & $9.28 \%$ \\
\hline vomiting & 9 & $6.42 \%$ \\
\hline Ascites & 7 & $5.00 \%$ \\
\hline
\end{tabular}

Out of 140 patients, $87(62.14 \%)$ patients were referred for convulsions and $45.71 \%$ with high blood pressure. Headache was the main presenting complain. 10 patients of severe preeclampsia had episodes of convulsion later on.
Blurring of vision and epigastric pain were complained by 16 and 13 patients respectively. Generilised edema were noted in $12.14 \%$ women. Other presented with oliguria, vomiting $\&$ ascites.

Table 3 Pregnancy outcome, Perinatal outcome \& Maternal complication

\begin{tabular}{|c|c|c|}
\hline Mode of delivery & $\begin{array}{l}\text { Number } \\
\text { of cases }\end{array}$ & Percentage $(\%)$ \\
\hline vaginal & 54 & $38.57 \%$ \\
\hline LSCS & 69 & $49.28 \%$ \\
\hline Instrumental & 17 & $12.14 \%$ \\
\hline \multicolumn{3}{|l|}{ Perinatal Outcome } \\
\hline Total no of live birth & 106 & $75.71 \%$ \\
\hline IUFD & 24 & $17.14 \%$ \\
\hline STILLBIRTH & 12 & $8.57 \% \%$ \\
\hline LOW BIRTH WEIGHT & 67 & $47.85 \%$ \\
\hline LOW APGAR SCORE & 75 & $53.57 \%$ \\
\hline NICU Admission & 83 & $59.28 \%$ \\
\hline Neonatal death & 12 & $8.57 \%$ \\
\hline Perinatal mortality & 48 & $34.28 \%$ \\
\hline \multicolumn{3}{|l|}{ Maternal complication } \\
\hline Abruptio placenta & 11 & $7.85 \%$ \\
\hline $\mathrm{PPH}$ & 51 & $36.42 \%$ \\
\hline DIC & 19 & $13.57 \%$ \\
\hline Acute renal failure & 15 & $10.71 \%$ \\
\hline HELLP Syndrome & 5 & $3.57 \%$ \\
\hline Pulmonary edema & 4 & $2.85 \%$ \\
\hline Neurological deficit & 8 & $5.71 \%$ \\
\hline Visual disturbances & 7 & $5 \%$ \\
\hline Intracranial hemorrhage & & $2.14 \%$ \\
\hline
\end{tabular}

The mode of delivery was determined on the basis of general condition of mother, fetal age and condition. Induction of labor was done after assessing bishop score. Majority of patients underwent caesarean section $(49.28 \%)$ for failed induction $(46.3 \%)$ or fetal distress $(24.7 \%)$. Vaginal delivery occurred in $38.57 \%$. The mean delivery time observed from admission to delivery was 6.8 hours.

Out of 140 cases, 106 were live birth, 24 intrauterine deaths, and 12 still births. Low birth weight babies were $47.85 \%$ and nicu admission required in $59.28 \%$ cases. Perinatal mortality occurred in $34.28 \%$ cases.

Maternal complications included PPH in 51, abruption placenta in 11 and DIC in 19 .other complication were HELLP Syndrome, acute renal failure, pulmonary edema. There were $10(7.14 \%)$ cases of maternal death. 


\section{Discussion}

Total number of deliveries in the year 2019 was 5891 including normal delivery and LSCS, out of which 207 women were admitted with severe preeclampsia and eclampsia and the incidence is $3.51 \%$. Out of 207 patients 140 patients were enrolled in this study according to the inclusion and exclusion criteria.

In this study $87(62.14 \%)$ patients were referred with convulsion and headache complained in $32.14 \%$ which is comparable with a study done by Singhal SR et al, 51\% patients presented with convulsion and $44 \%$ with headache..ref7

In this study majority of the patients were primigravida $53.57 \%$ and similar results were seen in studies done by Singhal et al and Shaikh et al.

LSCS was done $49.28 \%$ cases in this study which is similar to study done by Sibai ${ }^{9}$ and Inizi et a. Ref 10

Maternal complication included PPH in 51 patients followed by DIC, Abruptio placenta ,acute renal injury compared with study by Singhal et al[ ref 7] Out of 140 cases ,106 were live birth ,24 intrauterine death, and 12 still birth . Low birth weight babies were $47.85 \%$ and nicu admission required in $59.28 \%$ cases. Study by Shaikh $\mathrm{S}$ et al showed IUD in 14, neonatal death in 18 cases and perinatal loss of $38.6 \%{ }^{.8}$

\section{Conclusion}

Preeclampsia and eclampsia is a major cause of maternal morbidity and mortality in developing countries due to lack of antenatal care and poor access to health care facility. The incidence of eclampsia can be reduced by better antenatal care, early recognition of preeclampsia, adequate use of antihypertensive drugs and magnesium sulphate Delayed referral to higher centre will lead to irreversible changes with end organ damage resulting in increasing rate of maternal mortality and morbidity. Timed delivery improves maternal and perinatal outcome.

\section{References}

1. Say L, Chou D, Gemmill A, Tuncalp O, Moller AB, Daniels $\mathrm{J}$ et al.Global causes of maternal death: a WHO systemic analysis. Lancet Glob Health.2014;2 :e323-33.

2. Knight HE, Self A, Kennedy SH. Why are women dying when they reach hospital on time? A systematic review of the" Third Delay "PLoS One 2013 ;8:e63846.

3. Arora R, Ganguli RP, Swain S, Oumachigui A, Rajaram P . Determinants of maternal mortality in eclampsia in India.Aust $\mathrm{N} \mathrm{Z} \mathrm{J}$ Obstet Gynecol . 1994 ; 34 : 537-9 .

4. Roberts JM. Endothelial dysfunction in preeclampsia. Semin Reprod Endocrinol. 1998;16:5-15.

5. Sibai BM. Diagnosis, prevention, and management of eclampsia.Obstet Gynecol. 2005;105(2):402-10 .

6. Alvarez Navascues R, Marin R. Severe maternal complications associated with preeclampsia: an almost forgotten pathology? Nefrologia.2001;21(6):565-73.

7. Singhal S, Deepika, Anshu, Nanda S. Maternal and perinatal outcome in severe preeclampsia and eclampsia. South Asian Federation of Obstetrics and Gynecology .2009; 1(3): 25-8.

8. Shaikh S, Ruby AJ, Piotrowski M.Pre eclampsia related chorioretinopathy with Purtscher 's like findings and macular ischemia Retina $2003: 23: 247-50$.

9. Sibai BM .Eclampsia vi .Maternal perinatal outcome in 254 consecutive cases.Am j Obstet Gynecol 1990:163: 1049-55.

10. Al Inizi ST, Sharma H, Ahmed B. Eclampsia in Qutar .Maternal and fetal outcome and possible preventive measures. Middle East $\mathbf{J}$ Emerg Med 2005:5:1-9. 